\title{
Structural Insight into Allosteric Inhibition of Mycobacterium tuberculosis
}

\section{Tryptophan Synthase}

\author{
Andrzej Joachimiak ${ }^{1,6}$, Samantha Wellington ${ }^{2,3,4}$, Partha P. Nag ${ }^{2}$, \\ Robert Jedrzejczak $^{1}$, Natalia I. Maltseva ${ }^{1}$, Stewart L. Fisher ${ }^{2}$, Stuart L. Schreiber ${ }^{2,5}$, \\ Deborah T. Hung ${ }^{2,3,4}$, Karolina Michalska ${ }^{1}$ \\ ${ }^{1}$ Center for Structural Genomics of Infectious Diseases, University of Chicago, Chicago, USA \\ ${ }^{2}$ Broad Institute of MIT and Harvard, Cambridge, USA \\ ${ }^{3}$ Dep. of Microbiology and Immunobiology, Harvard Medical School, Boston, USA \\ ${ }^{4}$ Dep. of Molecular Biology and Center for Computational and Integrative Biology, Massachusetts \\ General Hospital, Boston, USA \\ ${ }^{5}$ Dep. of Chemistry and Chemical Biology, Harvard University, Cambridge, USA \\ ${ }^{6}$ Dep. of Biochemistry and Molecular Biology, University of Chicago, Chicago, USA
}

The rise of antibiotic-resistant pathogens requires redirecting drug discovery effort toward new cellular targets to provide new treatment options. Such alternative, yet unexplored opportunities exist in central metabolism, where many enzymes are only conditionally essential and thus their successful exploitation requires understanding of metabolic fluxes and pathway regulations under certain environmental conditions. In Mycobacterium tuberculosis, tryptophan biosynthesis represents one such promising pathway that could be targeted. In Mtb, the multistep L-Trp synthesis involves several enzymes: TrpEG, $\operatorname{TrpD}, \operatorname{TrpC}$ and $\operatorname{TrpAB}$, which is validated as conditionally essential in vivo. The whole-cell screening of a diversity-oriented synthetic library identified a novel bactericidal azetidine derivative, BRD4592, that specifically inhibits TrpAB (tryptophan synthase). TrpAB is a heterotetrameric, PLP-dependent complex catalyzing two last reactions in L-Trp synthesis: the conversion of indole-3-glycerol phosphate to indole and glyceraldehyde 3-phosphate (TrpA) followed by indole and L-Ser condensation to yield L-Trp (TrpB). During the catalytic cycle, indole is transferred from the TrpA to TrpB active site through $25 \AA$ hydrophobic channel to react with the L-Ser-PLP adduct. The enzymatic activities and substrate channeling are coordinated by complex allosteric communication between the subunits. This regulation involves cycling between various conformational states, with both subunits adopting either an open (inactive) or closed (active) state. Detailed kinetic, biophysical and structural evidence reveals that BRD4592 is an allosteric, mixed-type inhibitor, which binds specifically to a novel site within the intersubunit channel, at the $\operatorname{TrpA} \operatorname{TrpB}$ interface. The molecule promotes closed, active state of the TrpB subunit and increases enzyme affinity for the L-Ser substrate. BRD4592 acts on three levels: it limits flexibility of the interface potentially disrupting mutual activation of the subunits, prevents indole translocation, and increases product inhibition providing means to successfully inhibit both, $\operatorname{TrpA}$ and $\operatorname{TrpB}$ reactions. Our results indicate that allosteric binders may represent an effective tool to deactivate central metabolism enzymes, which often are allosterically regulated and might be predisposed for this type of inhibition.

This work was supported by the Broad Institute Tuberculosis donor group, the Pershing Square Foundation, the Bill \& Melinda Gates Foundation under grant OPP1032518, the National Institute of Allergy and Infectious Diseases, National Institutes of Health, Department of Health and Human Services, under contracts HHSN272200700058C and HHSN272201200026C and the U. S. Department of Energy, Office of Biological and Environmental Research, under contract DEAC02-06CH11357. For this work, University of Chicago is a subgrantee of Structural Genomics Consortium under a Bill \& Melinda Gates Foundation grant for the Structure-Guided Drug Discovery Coalition. 